\title{
Dynamic Windage Yaw Angle and Dynamic Wind Load Factor of a Suspension Insulator String
}

\author{
Shuang Zhao $\mathbb{D}^{1,2}$, Jiahao Yue $\mathbb{D}^{1},{ }^{1}$ Eric Savory, ${ }^{3}$ Zhitao Yan $\mathbb{D}^{1,2}$ Jiahao Chen $\left(\mathbb{D},{ }^{1}\right.$ \\ Bin Zhang $\mathbb{D},{ }^{4}$ and Liuliu Peng $\mathbb{D}^{5}$ \\ ${ }^{1}$ School of Civil Engineering and Architecture, Chongqing University of Science and Technology, Chongqing 401331, China \\ ${ }^{2}$ Chongqing Key Laboratory of Energy Engineering Mechanics \& Disaster Prevention and Mitigation, Chongqing 401331, China \\ ${ }^{3}$ Department of Mechanical and Materials Engineering, University of Western Ontario, London N6A 5B9, Canada \\ ${ }^{4}$ CMCU Engineering Co., Ltd.,, No. 17 Yu Zhou Road, Chongqing 400039, China \\ ${ }^{5}$ School of Civil Engineering, Chongqing University, Chongqing 400044, China \\ Correspondence should be addressed to Zhitao Yan; yzt@cqust.edu.cn
}

Received 29 July 2021; Revised 27 December 2021; Accepted 17 January 2022; Published 15 February 2022

Academic Editor: Denis Benasciutti

Copyright $\odot 2022$ Shuang Zhao et al. This is an open access article distributed under the Creative Commons Attribution License, which permits unrestricted use, distribution, and reproduction in any medium, provided the original work is properly cited.

\begin{abstract}
A simplified calculation method is proposed for determining the peak dynamic windage yaw angle $(\widehat{\varphi})$ of electricity transmission line (TL) tower suspension insulator strings (SISs). According to the rigid-body rule, the geometric stiffness matrix in the calculation of the windage yaw angle $(\varphi)$ of SISs is dominated by the average wind loads, while the fluctuating wind loads are the dominant factor in the elastic stiffness. With the average wind state of conductors as the initial calculation condition, the loadresponse-correlation (LRC) method can be used to determine the fluctuating windage yaw angle $\left(\varphi_{d}\right)$ and the corresponding equivalent static wind loads (ESWLs). Then, the improved rigid straight rod model, which uses the actual length of conductors rather than the projected length, was used to determine the average windage yaw angle $\bar{\varphi}$. Through the linear superposition of the horizontal increments of $\bar{\varphi}$ and $\widehat{\varphi}_{d}$ (the peak value of $\varphi_{d}$ ), the formulae to calculate the $\widehat{\varphi}$ of SISs were derived. Additionally, the formulae for the dynamic wind load factor, $\beta_{c}$, which is a key factor in designing wind loads for $\varphi$, were derived according to the principle of ESWLs, rather than being empirically determined by the Chinese code. Thus, the calculation model regarding the loads and response for the $\varphi$ of SISs was established, and an actual TL was used to verify the established calculation model. Afterward, the influence of the different engineering design parameters on $\varphi$ and its $\beta_{c}$ were analyzed. The parameter analyses show that the wind speed, span, and ground roughness influence the magnitudes of $\widehat{\varphi}$ and $\beta_{c}$, however, the height difference between the two suspension points of the conductors, the nominal height, and the sag-to-span ratio may be neglected in the approximate calculation. Our method offers a new solution to TL design when there are large deformations and small strains.
\end{abstract}

\section{Introduction}

Flashover accidents caused by the windage yaw of insulator strings damage transmission lines (TLs), leading to potential large-scale power failure. These accidents can occur when the wind speed is lower than the design wind speed. Therefore, the windage yaw design scheme for suspension insulator strings (SISs) needs to be improved to increase the reliability of the design.

The wind loads acting on SISs, especially those from the conductors, result in the windage yaw of SISs. Determining the design wind loads of conductors (termed the input problem) and the calculation method of the windage yaw angle, $\varphi$ (termed the output problem), is important for the calculation of the peak dynamic windage yaw angle, $\hat{\varphi}$, of SISs. In terms of the input problem, closed-form expressions have been derived for the wind-induced dynamic tension in overhead power TLs [1]. Then, by simplifying the derived root-mean-square (RMS) dynamic tension response spectrum, a calculation formula for the practical design of wind loads was provided [2]. Nonetheless, the background response of conductors was determined by Wang and Li [2] for 
using only a few modes, which inevitably introduces errors caused by modal truncation, leading to safety concerns. In China, when calculating $\hat{\varphi}$, the design wind loads for conductors and insulators in the Chinese technical code for the design of overhead TLs [3] are usually adopted. In the technical code, the design wind loads for conductors are expressed as average wind loads multiplied by the wind pressure nonuniformity factor, $\alpha$, which allows for the nonuniform distribution of the average wind in the span direction, whilst the dynamic wind load factor, $\beta_{c}$, allows for the action of the fluctuating wind. However, further research [4] found that, in some cases, the value of $\alpha \beta_{c}$ is less than unity, whereas the design wind loads of conductors in other countries $[5,6]$ are greater than the average loads. The dynamic magnification effect of the fluctuating wind in the wind yaw calculation cannot be ignored [7]. Furthermore, the microclimate and microterrain factors affect the wind loads for windage yaw calculation [8]. Under rain and wind conditions, negative aerodynamic damping can occur, and the peak swing amplitude of overhead conductors is larger than that under the wind alone, such that rain loads cannot be neglected [9]. Wind loads have been obtained using a two-way fluid-structure interaction simulation by considering the local mountainous terrain and coastal typhoon meteorological conditions, and it was found that the complex wind field has a non-negligible influence on the $\varphi$ of insulator strings [10]. Regarding the output problem, so far, the rigid straight rod (RSR) model is widely used as a simplified calculation method $[11,12]$. The method regards the SIS as a rigid straight rod with one end connected to the fixed hinge support at the tower. Then, $\varphi$ is determined by applying the equivalent loads from the conductors and SISs to the free end [13]. By comparing with the time-domain results of a finite element model (FEM), it was found that the traditional RSR model is not suitable for determining the dynamic peak response [14]. By considering the shielding effects from bundled conductors and the fluctuating wind effect, a correction formula was provided for the RSR method based on numerical calculations [15]. In a study of the deformation characteristics of the windage yaw of V-type composite insulators, it was found that the included angle of the V-type string is usually small because of the compression of the composite insulator string on the leeward side [16].

These studies helped to develop calculation methods for the $\varphi$ of insulator strings. However, because of the improper simplifications and the lack of long-term testing, these methods have not been widely used in engineering design. The main issue with the existing windage yaw calculation is the conflict between the nonlinear characteristics of the wind-induced vibration of the lightweight and flexible conductors and the linear wind-induced vibration calculation theory. Yang $[17,18]$ proposed the theory and algorithm of the rigid-body rule, which has been successfully used to deal with the large deformation and small strain of a truss structure, frame structure, and plate shell structure. However, the concept of the rigid-body rule can also be effectively used to solve the problem of a cable structure, such as the windage yaw of TLs. Here, the windage yaw is dominated by rigid-body displacement and can be solved by decomposing it into a rigid-body displacement and natural deformation. Thus, the fluctuating windage yaw can effectively be solved by frequency-domain calculation methods, such as the gust load factor (GLF) method [19], the load-response-correlation (LRC) method [20], and effective static load distribution methods [21], which apply to linear structures, whereas the average windage yaw, dependent on the average wind, can be easily solved by static calculations. Although research has been conducted to address this issue $[1,22]$, the decomposition of the response has lacked a reasonable basis. The mode decomposition method with finite modes was inappropriately used to solve the wind-induced vibration of conductors with dense frequencies of each order, and the RSR method was not improved.

In this work, we propose a systematic and simplified calculation method for the $\hat{\varphi}$ of SISs in the along-wind direction because of the buffeting excited by synoptic wind (that produces Gaussian or weakly non-Gaussian wind loads). Theoretically, this new method offers simplified calculations for the wind-resistant design of TLs considering the influence of windage yaw. The geometric stiffness under the action of the average wind and self-weight is taken as the initial calculation condition, and the equivalent static wind loads (ESWLs) for $\hat{\varphi}$ are determined based on the LRC method. A more accurate RSR model is proposed to calculate the average windage yaw angle $(\bar{\varphi})$ of the SISs, based on the force balance and triangle principle by taking into account the actual length of the conductors. The peak fluctuating windage yaw angle $\left(\widehat{\varphi}_{d}\right)$ of the SISs is determined by the frequency-domain calculation. Then, $\widehat{\varphi}$ is obtained by the linear superposition of the horizontal increments of $\bar{\varphi}$ and $\widehat{\varphi}_{d}$. Additionally, the dynamic wind load factor, $\beta_{c}$, which is a key factor in the design wind loads for $\varphi$, is determined according to the principle of ESWLs. Afterward, the proposed windage yaw calculation model, including loads and response, is verified by the time-domain results. Finally, the influence of important design parameters, such as the wind speed, the height difference between the two suspension points of the conductors (termed "height difference" hereafter), the nominal height, the sag-to-span ratio, the span, and the ground roughness on $\widehat{\varphi}$ and $\beta_{c}$ are analyzed using the proposed calculation model. It is anticipated that the results from the present work may be incorporated into the wind-resistant design of TLs.

\section{Calculation Methods}

2.1. Equivalent Static Wind Loads for Windage Yaw Angle. TLs have a catenary configuration under self-weight and exhibit geometrically nonlinear behavior under wind loads. Research has shown that transmission towers have little influence on the wind-induced vibration response of TLs [23]. To simplify the calculation of the $\varphi$ of the SIS, the influence of the tower is neglected, and the suspension point of the insulator string on the tower is regarded as fixed hinge support (point A in Figure 1). Additionally, since the study in this paper is within the framework of the Chinese code, when applying the present work in other countries and regions that use different TL design codes, the methodology 


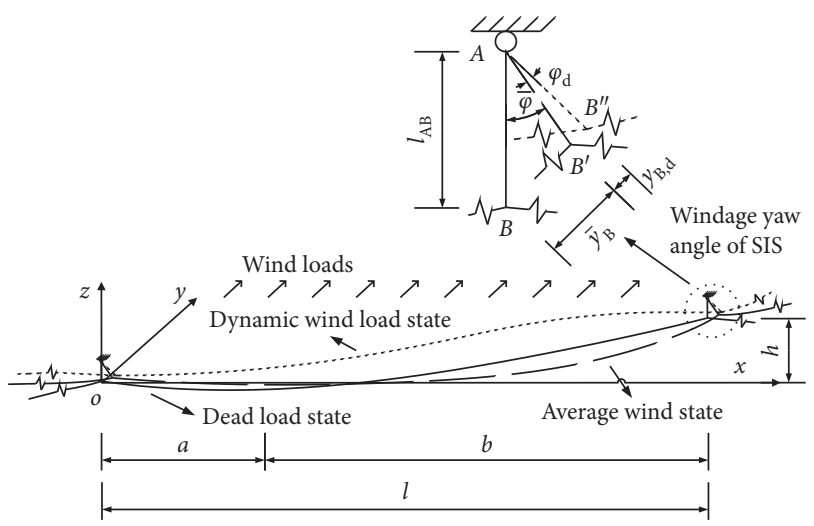

Figure 1: Calculation model of the windage yaw angle.

proposed in this paper should be used in relation to those national codes. The calculation model for $\varphi$ is shown in Figure 1, where $a$ is the horizontal distance from the origin of the coordinates to the lowest point of the conductors, $l$ represents the span, and $l=a+b, h$ is the height difference between the two suspension points of the conductors, $\bar{y}_{B}$ is the along-wind displacement of point $B$ under the average wind loads, and $\bar{y}_{B}=l_{\mathrm{AB}} \sin \bar{\varphi}, l_{\mathrm{AB}}$ is the length of the insulator string between point $\mathrm{A}$ and point $\mathrm{B}, y_{B, d}$ is the alongwind displacement of point $B$ under fluctuating wind loads, $B$ is the connection point between the conductor and the SIS, and the superscripts " " " and " " " of this point indicate the average wind state and the dynamic wind state, respectively.

Usually, the wind loads in the along-wind direction can be decomposed into two parts: the average wind loads and the fluctuating wind loads. The matrix expression of the conductor vibration in response to those loads is,

$$
\mathbf{M} \ddot{\mathbf{Y}}_{d}+\mathbf{C} \dot{\mathbf{Y}}_{d}+\mathbf{K}\left(\overline{\mathbf{Y}}+\mathbf{Y}_{d}\right)=\mathbf{L}\left(\overline{\mathbf{p}}+\mathbf{p}_{d}\right),
$$

where $M, C$, and $K$ are the mass, damping, and stiffness matrices, respectively. $L$ is the transformation matrix composed of the equivalent force at the nodes when unit wind pressure loads are applied to the subordinate areas of the nodes. $\overline{\mathbf{p}}$ and $\mathbf{p}_{d}$ are the average wind loads and fluctuating wind loads per unit area, respectively. Under the fluctuating wind loads, $\ddot{\mathbf{Y}}_{d}, \dot{\mathbf{Y}}_{d}$, and $\mathbf{Y}_{d}$ are the acceleration, speed, and displacement in the along-wind direction, respectively. Under the average wind loads, $\overline{\mathbf{Y}}$ is the displacement in the along-wind direction. Based on the quasistatic assumption, $\overline{\mathbf{p}}$ and $\mathbf{p}_{d}$ are expressed as follows:

$$
\begin{aligned}
\overline{\mathbf{p}} & =0.5 \rho_{a} C_{d} \overline{\mathbf{v}}^{2}, \\
\mathbf{p}_{d} & =\rho_{a} C_{d} \overline{\mathbf{v}} * \mathbf{v}^{\prime},
\end{aligned}
$$

where $\rho_{a}$ is the air density per unit volume, $\overline{\mathbf{v}}$ is the average wind speed, .* indicates that the corresponding elements of the front and rear matrices are multiplied, $\mathbf{v}^{\prime}$ is the fluctuating wind speed, and $C_{d}$ is the drag coefficient.

The conductor has a light and flexible structure; under strong wind loads, it can exhibit the following mechanical behavior: (1) the structure has large deformation and geometric nonlinearity, (2) there is a nonlinear relationship between the force and displacement of the structure, and (3) under dynamic wind loads, the structure has time-varying stiffness. Thus, equation (1) is a variable coefficient differential equation so that the linear superposition principle cannot be used to calculate the wind-induced response of the conductors. When conductors undergo large deformation, they are often in the elastic stage. However, if the equilibrium equation is established in the position before the deformation, according to the linear analysis method, the real mechanical behavior of a TL cannot be obtained. It is the key reason why frequency-domain calculation methods, such as the GLF method [19], LRC method [20], and the effective static load distribution method [21] cannot be used for reliable windage yaw calculation of TLs. $K$ includes the geometric stiffness matrix and elastic stiffness matrix [24]. The geometric stiffness matrix reflects the effect of the initial external force because of the change in member shape and position. The rigid-body concept can effectively deal with various large deformation and small strain problems $[17,18]$. According to this concept, the windage yaw of TLs is the superposition of two processes: the rigid-body displacement of the TL, which occurs from the dead load state to the average wind state and, consequently, the small amplitude and the back-and-forth motion, which occurs from the average wind state to the dynamic wind load state (as shown in Figure 1). In the first process, the element nodal force has the same rigid body displacement with the element, whose magnitude is unchanged, and this force balance is still maintained in the second process. Thus, the small amplitude motion of the second process is calculated after the end of the first process is set as an initial state. Hence, the first process end accounts for the main part of the windage yaw and determines the geometric stiffness matrix, whereas the second process affects only the elastic stiffness matrix. By the decomposition of the windage yaw, the static calculations and the frequency-domain method can be used to solve the first and second processes, respectively. The above description can be summarized as follows: under the action of fluctuating wind loads, the motion of the conductor is regarded as small displacement, and the load-response relationship is almost linear. The stiffness matrix is consistent with that under the average wind state, $\mathbf{K}_{\bar{Y}}$, i.e., $\mathbf{K}=\mathbf{K}_{\bar{Y}}$.

Therefore, under the action of fluctuating wind loads, the matrix expression for the vibration of the conductors is as follows:

$$
\mathbf{M} \ddot{\mathbf{Y}}_{d}+\mathbf{C} \dot{\mathbf{Y}}_{d}+\mathbf{K}_{\bar{Y}} \mathbf{Y}_{d}=\mathbf{L} \mathbf{p}_{d} .
$$

Unlike equation (1), equation (4) is a differential equation with constant coefficients and can be calculated by the linear superposition principle. The average response of the conductors is regarded as a static response, and the average displacement of the conductors can be expressed by the nonlinear static balance equation, which is as follows:

$$
\mathbf{K}_{\bar{Y}} \overline{\mathbf{Y}}=\mathbf{L} \overline{\mathbf{p}} \text {. }
$$

Because of the large aerodynamic damping of the conductors, the resonant response is negligible, and hence, the fluctuating response is dominated by the quasi-static 
background response $[25,26]$. The resonant response of the conductors is also neglected in the ASCE code [5]. Similarly, regardless of the resonant response, the ESWLs for equation (4) are, herein, calculated by the LRC method. Thus, the background response, $\mathbf{Y}_{b}$, of the conductors at the node can be expressed as follows:

$$
\mathbf{K} \mathbf{Y}_{b}=\mathbf{L p}_{d} .
$$

The variance of $\mathbf{Y}_{b}$ can be expressed as follows:

$$
\boldsymbol{\sigma}_{b}^{2}=\operatorname{diag}\left(\mathbf{Y}_{P} \int_{-\infty}^{+\infty} \mathbf{S}_{p p} \mathbf{d} \omega \mathbf{Y}_{P}^{T}\right)
$$

where diag is the new matrix formed by the diagonal elements of the matrix in parentheses, $Y_{\mathrm{P}}$ is the solution of the static balance equation, $\mathbf{K Y}_{P}=\mathbf{L}$, and $\mathbf{S}_{p p}$ is the crossspectrum matrix of the fluctuating wind pressure.

The correlation coefficient between the fluctuating load effect and the fluctuating wind loads is as follows:

$$
\begin{gathered}
\boldsymbol{\rho}_{\operatorname{Pr}}=\frac{\overline{\mathbf{L} \mathbf{p}_{d} \mathbf{r}^{T}}}{\left(\mathbf{L} \boldsymbol{\sigma}_{p} \boldsymbol{\sigma}_{b}^{T}\right)}, \\
\mathbf{r}=\mathbf{I}_{r}^{T} \mathbf{L} \mathbf{p}_{d},
\end{gathered}
$$

where the overbar indicates a time average, ./ indicates the division of the corresponding elements of the front and rear matrices, and $\boldsymbol{\sigma}_{p}$ is the standard deviation of $\mathbf{p}_{d}$. $\mathbf{I}_{r}$ denotes the influence function for the load effect, $r$.

The distributions of the ESWLs per unit area of the nodes are as follows:

$$
\begin{aligned}
\widehat{\mathbf{p}}(:, i) & =\overline{\mathbf{p}}+\widehat{\mathbf{p}}_{b}(:, i), \\
\widehat{\mathbf{p}}_{b}(:, i) & =g \boldsymbol{\rho}_{\operatorname{Pr}}(:, i) . * \boldsymbol{\sigma}_{p},
\end{aligned}
$$

where $(:, i)$ are all the elements in the $i$ column of the matrix, and $g$ is the background peak factor. Equation (10) is the general formula that can be used to calculate the ESWLs at different locations and the different responses of the conductors. In Figure 1 , point B is selected as the calculation target point. When the along-wind displacement, $y_{B}$, of the target point reaches its maximum value, the $\varphi$ of the SIS reaches the maximum $\widehat{\varphi}$. According to equations (7)-(10), $\widehat{\varphi}$ and its ESWLs can be calculated using $y_{B}$ as the load effect.

\subsection{Dynamic Peak Windage Yaw Angle. As shown in Fig-} ure 1, under the dead load state, the SIS, which is regarded as a rigid straight rod, is located between the points $A$ and $B$. Under the average wind state, point $B$ moves to point $\mathrm{B}^{\prime}$ in the along-wind direction, and the increment of $\varphi$ is $\bar{\varphi}$. With the addition of the fluctuating wind, point $\mathrm{B}^{\prime}$ moves to point $\mathrm{B}^{\prime \prime}$ in the along-wind direction, and the increment of $\varphi$ is $\varphi_{d}$. The dynamic peak windage yaw angle, $\hat{\varphi}$, is decomposed into $\bar{\varphi}$ and $\widehat{\varphi}_{d}$ (the peak value of $\varphi_{d}$ ). The RSR model is widely used to simplify calculations for the $\varphi$ of SISs $[11,12]$. However, the applicability of this model is limited. Large errors may occur when the layout and horizontal loads of a TL are nonuniform along the span direction, e.g., the TL that has height differences between the two conductor suspension points and experience fluctuating wind loads [14, 15]. The analysis presented in this section improves the traditional RSR model and uses the improved model and the frequency-domain method to calculate $\bar{\varphi}$ and $\widehat{\varphi}_{d}$, respectively. Based on the force balance and triangle principle, $\bar{\varphi}$ can be derived from the following:

$$
\bar{\varphi}=\arctan \left(\frac{0.5 \bar{G}_{h}+\bar{W}_{h}}{0.5 G_{v}+W_{v}}\right),
$$

where $\bar{G}_{h}$ and $G_{v}$ are, respectively, the average wind loads and the weight at the centroid of the SIS, whereas $\bar{W}_{h}$ and $W_{v}$ are the horizontal loads and vertical loads at point $\mathrm{B}^{\prime}$ of the SIS, which are generated by the average wind loads and the weight of the conductors, respectively. The expressions of $\bar{W}_{h}$ and $W_{v}$ can be written as follows:

$$
\begin{aligned}
& \bar{W}_{h}=\bar{P}_{h} \Gamma_{h}, \\
& W_{v}=P_{v} \Gamma_{v},
\end{aligned}
$$

where $\bar{P}_{h}$ and $P_{v}$ are the horizontal and vertical loads per unit length of the conductors, respectively, $\Gamma_{h}$ is the total alongconductor length between the two half-span points $(l / 2)$ on either side of the tower, and $\Gamma_{v}$ is the total along-conductor length between the two lowest conductor points on either side of the tower. The increment of the conductor length caused by the wind loads is very small compared with its total length, and hence, $\Gamma_{h}$ and $\Gamma_{v}$ can be determined under a dead load state. According to the coordinate system established in Figure 1, the expression of the catenary equation of the conductor is as follows:

$$
\begin{aligned}
& z=\frac{2 \sigma_{0}}{\gamma} \operatorname{sh} \frac{\gamma x}{2 \sigma_{0}} \operatorname{sh} \frac{\gamma(x-2 a)}{2 \sigma_{0}}, \\
& a=\frac{l}{2}-\frac{\sigma_{0}}{\gamma} \operatorname{arcsh} \frac{h}{\left(2 \sigma_{0} / \gamma\right) \operatorname{sh}\left(\gamma l / 2 \sigma_{0}\right)}, \\
& \gamma=\frac{G_{0}}{A_{s}},
\end{aligned}
$$

where $\sigma_{0}$ is the initial horizontal conductor stress. When the target point is higher than the other suspension point, $h$ is positive. $G_{0}$ and $A_{s}$ are, respectively, the self-weight per unit length and the cross-sectional area of the conductors. Using equation (15), $\Gamma_{h}$ and $\Gamma_{v}$ can be calculated. Equations (13)-(17) are closed-form formulations that reduce the calculation error of the windage yaw angle compared with the traditional RSR method and are suitable for different cases, especially those with height differences. follows:

Based on the triangle principle, the expression for $\widehat{\varphi}$ is as

$$
\begin{aligned}
& \widehat{\varphi}=\arcsin \frac{\widehat{y}_{B}}{l_{A B}}, \\
& \widehat{y}_{B}=\bar{y}_{B}+\widehat{y}_{B, d},
\end{aligned}
$$


where $\hat{y}_{B, d}$ is the peak along-wind displacement of point B under fluctuating wind loads and can be calculated by the linear superposition principle. Thus, the expression of $\hat{y}_{B, d}$ is as follows:

$$
\widehat{y}_{B, d}=g\left(\boldsymbol{\rho}_{P y_{B}} * \boldsymbol{\sigma}_{p}\right)^{T} \mathbf{L}^{T} \mathbf{I}_{y_{B}}
$$

where $\rho_{P y_{B}}$ is the correlation coefficient of the fluctuating wind loads and $y_{B}$, and $\mathbf{I}_{y_{B}}$ is the influence function of $y_{B}$. Thus, the calculation model of $\hat{\varphi}$ is established by equations (7)-(20).

\subsection{Dynamic Wind Load Factor of the Windage Yaw Angle.} The expression for the design wind loads for conductors in the Chinese technical code for the design of overhead TLs [27] is as follows:

$$
W_{x}=\alpha \beta_{c} \overline{W_{x}}=\alpha w_{0} \mu_{z} \mu_{s c} \beta_{c} d L_{p} B_{i} \sin ^{2} \theta,
$$

where $\bar{W}_{x}$ is the average wind loads, $w_{0}$ is the wind pressure at a reference height of $10 \mathrm{~m}, \mu_{z}$ is the wind pressure height variation coefficient, $\mu_{s c}$ is the drag coefficient, $d$ is the outer diameter of the conductors, $B_{\mathrm{i}}$ is the amplification factor for the wind loads considering any ice-coverage, and $\theta$ is the angle between the along-wind direction and the extension direction of the conductors. Moreover, using Table 10.1.18-1 [27] in this design code to calculate the windage yaw, the value of $\alpha$ is less than unity and related only to the wind speed, and $\beta_{c}$ is set to unity. Since $\alpha$ and $\beta_{c}$ of this design code are determined empirically, $W_{x}$ is less than $\bar{W}_{x}$, which contradicts the concept of ESWLs.

The physical meaning of $\beta_{c}$ is consistent with that of $\beta$ in the Chinese load code [28], both of which consider the dynamic effect caused by the fluctuating wind. According to the concept of ESWLs, $\boldsymbol{\beta}_{c}=\widehat{\mathbf{p}} . / \overline{\mathbf{p}}, \boldsymbol{\beta}_{c}$ calculated by the LRC method is not a constant. $\beta_{c}$ is averaged according to the distribution characteristics of $\widehat{\mathbf{p}}$ to give a value that is then considered to be uniformly distributed, which facilitates the design. $\widehat{\mathbf{p}}$ has its maximum value at a selected target point, and it approaches $\overline{\mathbf{p}}$ far away from the target point. Both $\widehat{\mathbf{p}}$ and $\overline{\mathbf{p}}$ are nonuniform. Therefore, a calculation range can be set artificially. Within this calculation range, the ESWLs at the target point can be calculated by averaging. For example, when there is no height difference between the target point and the conductor suspension point of the adjacent tower, the left and right half-spans of the target point can be set as the calculation range. Moreover, when there is a height difference between the target point and the conductor suspension point of the adjacent tower, since the ESWLs at the target point are larger in magnitude, the left and right quarter spans of the target point are selected as its calculation range. Under the calculation range, assuming a uniform distribution, $\beta_{\mathrm{c}}$, can be calculated using the following:

$$
\begin{gathered}
\beta_{c}=\frac{\widehat{q}}{\bar{q}}=1+\frac{\widehat{q}_{b}}{\bar{q}}, \\
\widehat{q}_{b}=\frac{\sum \widehat{\mathbf{p}}_{b}}{\sum \Gamma},
\end{gathered}
$$

$$
\bar{q}=\frac{\sum \overline{\mathbf{p}}}{\sum \Gamma}
$$

where $\sum$ indicates the sum of elements in the calculation range, and $\sum \Gamma$ is the actual length of the conductors in the calculation range. Equations (22)-(24) have been verified by the present authors (currently unpublished) for several different TLs and the relative errors of $\widehat{\varphi}$ from FEM, and the proposed model is less than $\pm 6 \%$. Hence, $\beta_{c}$ can be easily obtained with sufficient precision for engineering applications. Thus, the calculation model of the dynamic wind load factor is established using equations (22)-(24), and the value of $\beta_{c}$ calculated by this calculation model meets the concept of ESWLs and incorporates the influence of various design parameters. Therefore, this formulation for $\beta_{c}$ is more accurate and has a sounder physical basis than that specified in the Chinese technical code for the design of overhead TLs [27]. The design wind loads of the windage yaw angle can be calculated by substituting $\beta_{\mathrm{c}}$ into equation (21). Some example calculations are presented in the next section.

When determining the windage yaw of SISs, equation (21) is used to calculate the design wind loads, and equation (12) is used to calculate the responses in the existing methods. For complex types of transmission lines, such as those with large spans, large height differences, and significant dynamic effects, the time-domain analysis of finite element models is required to determine the windage yaw. The method proposed in this paper solves the problem of applying the LRC method to calculate the windage yaw and gives closed-form formulations of the dynamic wind loads of SISs. Considering the actual length of the conductors, the RSR model is improved by equations (13)-(17). A two-step method for calculating $\widehat{\varphi}$ is proposed. Firstly, the improved RSR model is used to determine $\bar{\varphi}$, and the LRC method is used to determine $\widehat{\varphi}_{d}$. Secondly, $\beta_{c}$ is determined based on the LRC method, and its nonuniform distribution is processed to an equivalent average distribution for engineering practice using equations (22)-(24). Compared with the existing methods, the proposed method not only enriches the application scenarios for calculating the windage yaw of SISs in the wind-resistant design codes but also has the advantage of efficient calculation (see the section below for details).

\section{Calculations}

3.1. Calculation Parameters. A $500-\mathrm{kV}$ TL, whose layout scheme is two tension towers at both ends, with three suspension towers between them, all arranged in a straight line, is selected, and the length between the two tension towers is $2.2 \mathrm{~km}$, as shown Figure 2. In Figure 2, $H$ represents the nominal height and is also equal to the height of the suspension point of the insulator string. Then, the connecting point of the insulator string and the conductor at suspension point 2 is arbitrarily selected as the target point for the calculation case to illustrate the methodology, although the calculation method is also applicable to any other selected point. Moreover, the conductors are type $4 \times$ JLHA1/G1A-575/40-45/7, whose physical parameters 


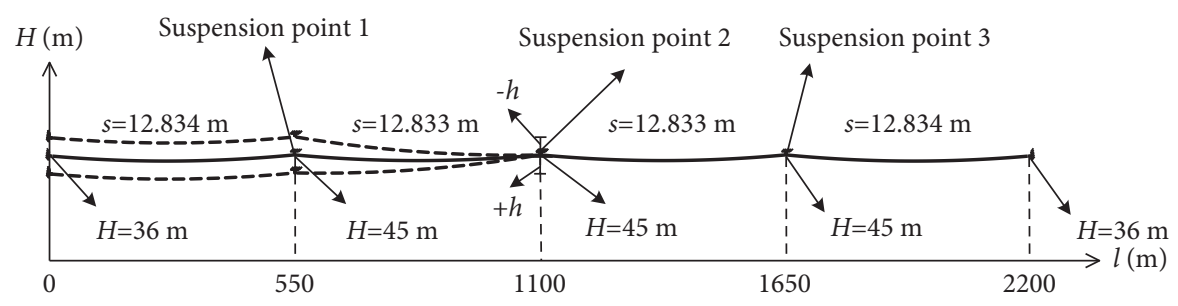

FIgURE 2: Calculation sketch of the transmission line spans.

are shown in Table 1. According to the Chinese technical code for the design of overhead TLs [27], the drag coefficient of the conductors is $C_{\mathrm{d}}=1.1$. The physical parameters of the SIS on the tension tower and the suspension tower are shown in Table 2. Meanwhile, the design average wind speed at $10 \mathrm{~m}$ height is $30 \mathrm{~m} / \mathrm{s}$, the oncoming wind direction is perpendicular to the span direction, and category B ground roughness (the terrain type of open country and towns with sparse houses) is used for the calculations. According to the Chinese load code [28], the peak factor is 2.5 .

3.2. Verification of Windage Yaw Angle Calculation. The proposed calculation model for the windage yaw of SISs in section 2 is verified by the FEM in this section. Before the verification, two simplified approaches need to be elaborated. Firstly, in this work, we focus on the overall motion of the conductors under wind loads, rather than the local subspan oscillation, and hence, the bundled conductors are combined into a single conductor according to the force equivalent. Secondly, $\alpha$ considers the nonuniform distribution of the average wind along the span direction, which represents the characteristics of the average wind. Here, we focus on the dynamic effect of the fluctuating wind. Therefore, $\alpha$ is not considered in the following analyses. Using equations (1)-(11), the ESWL per unit area of the conductors and its component distribution are determined, as shown in Figure 3. The components of the ESWLs are dominated by the average component, and the average component distribution is similar to the catenary shape under the dead load state. The background component reaches its peak at the target point and approaches zero at both ends. Because of the symmetry of the structural arrangement and the target point being located on the symmetry axis, the distributions of the ESWLs and their components have symmetry. The wind loads determined in Figure 3 are used to calculate the windage yaw angle and its $\beta_{\mathrm{c}}$. Using equations (12)-(14), we obtain $\bar{\varphi}=51.93^{\circ}, \bar{W}_{\mathrm{h}}$ $=60.35 \mathrm{kN}$, and $W_{v}=42.08 \mathrm{kN}$, and through equations (18) and (19), we derive $\widehat{\varphi}=58.53^{\circ}$ and $\widehat{y}_{B}=5.827 \mathrm{~m}$. Through equations (22)-(24), we calculate $\beta_{\mathrm{c}}=1.281, \widehat{q}_{b}=7.64 \mathrm{~N} / \mathrm{m}$ and $\bar{q}=27.24 \mathrm{~N} / \mathrm{m}$.

The correctness of the proposed calculation model is verified by the time-domain calculation results of the FEM. The harmonic wave superposition method [29] is used to simulate the turbulent wind fields for a Category B ground roughness [28]. Consistent with the Chinese load code [28], the power spectrum function of the height-independent fluctuating wind speed, proposed by Davenport [30], and the spatial correlation function, proposed by Shiotani and Arai [31], are adopted. The greater the number of wind speed samples $N$, the higher the accuracy of the wind speed simulation, however, this increases the calculation cost. When the fast Fourier transform algorithm is applied to the harmonic wave superposition method, the value of $N$ must be equal to an exponential function of 2 [29]. Herein, we set $N=4096$ and the cut-off frequency $f_{\text {up }}=4 \mathrm{~Hz}$ for the simulated wind speed. Then, the time interval is $\Delta t=1 /\left(2 f_{\text {up }}\right)=$ $0.125 \mathrm{~s}$, and the simulation duration is $1024 \mathrm{~s}$. Only the wind speeds within the height, from 24.228 to $38.168 \mathrm{~m}$, which are the lowest and highest heights of the conductors, respectively, of the conductors, are simulated. The characteristics of the simulated wind field for the average wind profile, the turbulence intensity profile, and the fluctuating wind speed power spectrum at $38.168 \mathrm{~m}$ of height are compared with those in the Chinese load code [28], as shown in Figure 4, where $I_{z}=\sigma_{v} / \bar{v}$ is the turbulence intensity, $\sigma_{v}$ is the standard deviation of $v^{\prime}, f$ is the frequency of the fluctuating wind speed, $S_{v}$ is the fluctuating wind speed power spectrum function, and the turbulence length scale, $L_{v}$, is $1200 \mathrm{~m}$ according to Davenport [30]. The difference between the results from the simulated wind field and the Chinese load code can be eliminated when $N$ is infinite. The maximum and minimum relative error of average wind speeds obtained from the Chinese load code compared to those obtained by the simulated wind field are $0.7 \%$ and $0.5 \%$, respectively. Similarly, the errors in the turbulence intensity are $4.4 \%$ and $-4.2 \%$, respectively. The comparison demonstrates that the simulated values are in good agreement with the calculated values from the Chinese load code.

The FEM was conducted using the ANSYS software [32], and the windage yaw of the TL was analyzed under the simulated wind field. The conductor and insulator string are simulated by the Link 180 element [33], whose element length is $10 \mathrm{~m}$ and component length, respectively. The element is a uniaxial tension-compression element with three degrees of freedom at each node, and a tension-only (for cable structures) setting is available, which can be used to model trusses, sagging cables, links, springs, and so on. The structural damping ratio is $0.5 \%$ according to LoredoSouza and Davenport [25]. Furthermore, aerodynamic damping and structural self-weight are taken into consideration. The initial operating tension is $55.39 \mathrm{kN}$ according to the actual design parameter. The conductor shape under the dead load state is determined by equations (15)-(17). The analysis type of the ANSYS software is set as transient analysis, and the transient effects and large-deflection effects 
TABLE 1: Physical parameters of the conductors (JLHA1/G1A-575/40-45/7 ACSR type).

\begin{tabular}{lccc}
\hline Cross-sectional area $\left(A_{\mathrm{s}}\right)\left(\mathrm{mm}^{2}\right)$ & Elastic modulus $(\mathrm{MPa})$ & Mass per unit length $(\mathrm{kg} / \mathrm{km})$ & Outer diameter $(\mathrm{mm})$ \\
\hline 621 & 63000 & 1917 & 32.40
\end{tabular}

TABLE 2: Physical parameters of the insulator string.

\begin{tabular}{lcccc}
\hline Position & Length $\left(l_{\mathrm{AB}}\right)(\mathrm{m})$ & Elastic modulus $(\mathrm{MPa})$ & Mass $(\mathrm{kg})$ & Wind-shielding area $\left(\mathrm{mm}{ }^{2}\right)$ \\
\hline Tension tower & 8.33 & 72000 & 1614.60 & 113400 \\
Suspension tower & 6.83 & 72000 & 1238.08 & 101800 \\
\hline
\end{tabular}

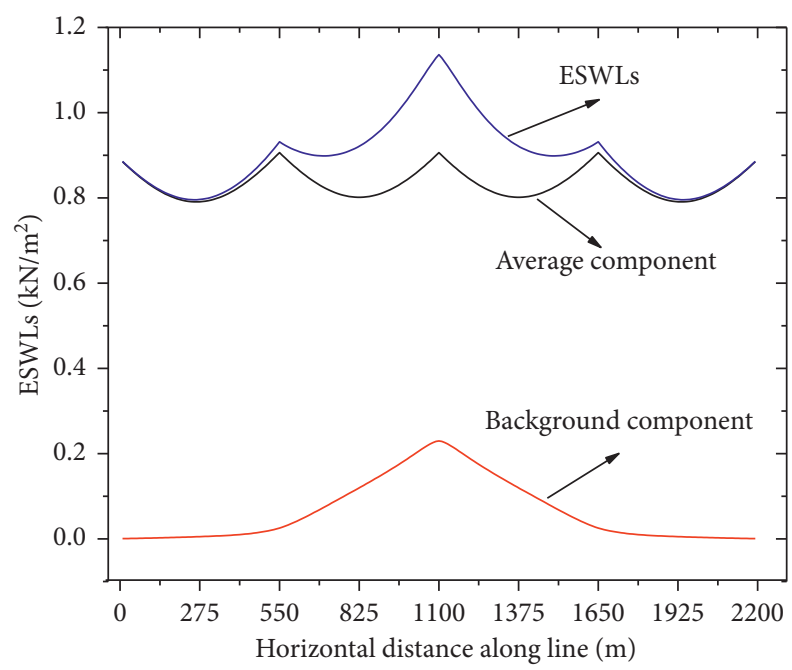

Figure 3: Equivalent static wind loads and the distributions of their components.

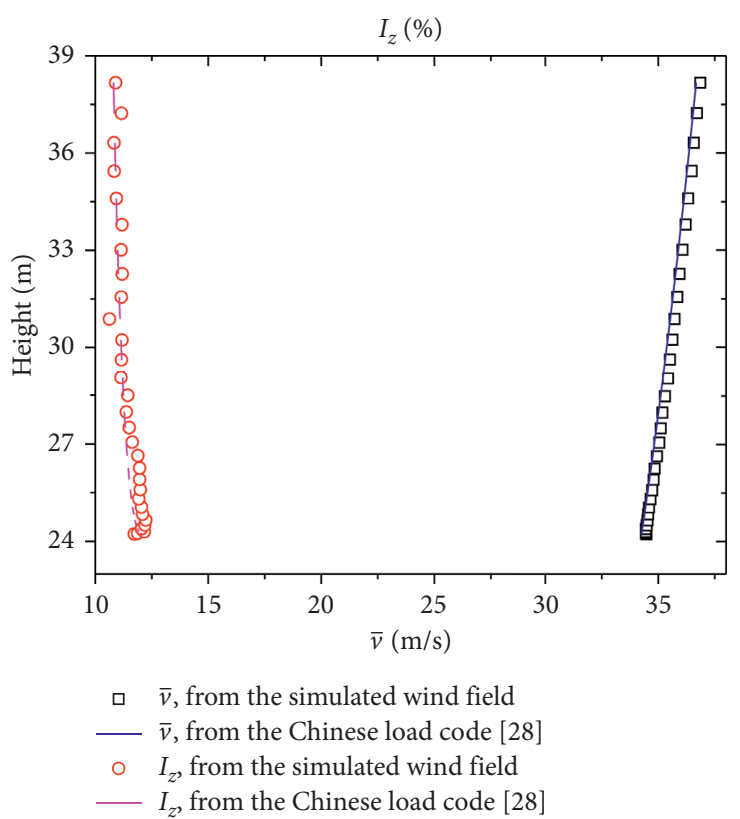

(a)

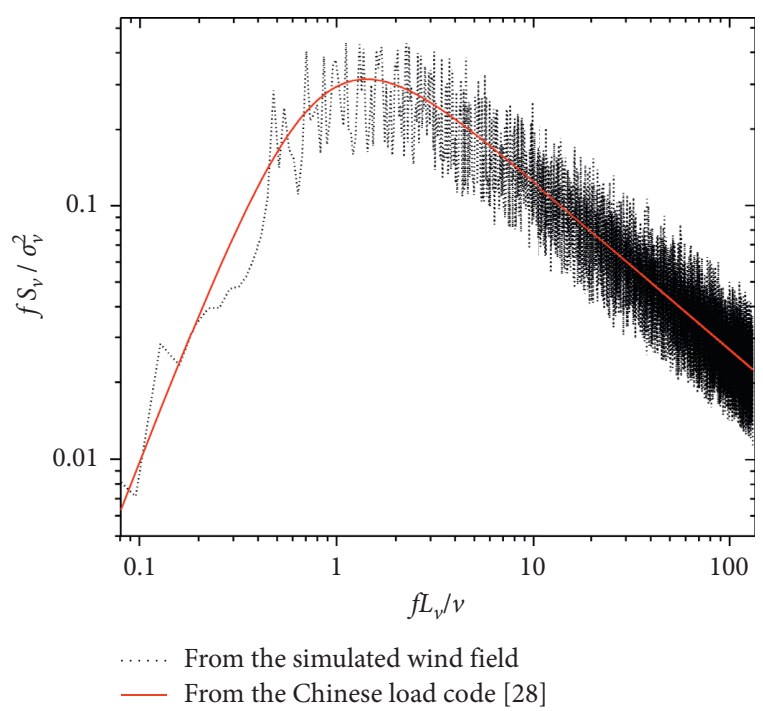

(b)

Figure 4: Comparison of the simulated values and the calculated values of the Chinese load code for the wind field characteristics for Category B ground roughness (GB 50009-2012, 2012). (a) Average wind speed $(\bar{v})$ profile and turbulence intensity $\left(I_{z}\right)$ profile. (b) Nondimensional wind speed spectrum at $38.168 \mathrm{~m}$ height. 
options are turned on. The wind loads of the component elements are applied to its element node equivalently. The restraint mode of the connection between the insulator string and the tower, the physical parameters and the geometric information of the conductors, and the insulator strings are consistent with those in Figure 2 and Tables 1 and 2. Then, the established FEM (as shown in Figure 5) is used for conducting time-domain calculations. In Figure 5, the directions of the $x, y$, and $z$ axes are parallel to the crossconductor, along-conductor, and vertical directions, respectively. In addition, the accuracy of the time-domain calculation has been verified [34].

In the time-domain calculation, the $\bar{\varphi}$ and $\widehat{\varphi}$ of the target point are $50.86^{\circ}$ and $56.23^{\circ}$, respectively. The relative error of $\bar{\varphi}$ and $\hat{\varphi}$ obtained from the time-domain calculation compared to those obtained by the proposed calculation model are $2.1 \%$ and $4.1 \%$, respectively. Hence, the results of the calculation model are in agreement with those of the timedomain model and can provide sufficient precision in engineering applications. Moreover, the computational times of these results are approximately $3 \mathrm{~s}$ and $2 \mathrm{~h}$, respectively, showing that the present calculation model is computationally much more efficient.

\section{Analyses of Important Influencing Parameters}

The main variables that influence the value of $\beta_{c}$ in (22) are the wind speed, the conductor height, the spatial correlation, and the turbulence. These variables can be further subdivided into various influence parameters, such as the height difference, the nominal height, the sag-to-span ratio, the span, and the ground roughness. The codependency of the influence parameters is neglected herein. Taking Figure 2 as an example, the influence parameters are changed independently within the design range, and the proposed calculation model is used to assess their effects research on $\bar{\varphi}, \widehat{\varphi}$, and $\beta_{c}$. In practical engineering design, many conductor and SIS types are used, although only one type (as shown in Tables 1 and 2) of each is analyzed in this work. For other types of conductors and SISs, the method proposed in this paper can be used to calculate the windage yaw of the TLs.

4.1. Influence of Wind Speed. The design average wind speed at the reference height of $10 \mathrm{~m}, \bar{v}_{10}$, is varied from $10 \mathrm{~m} / \mathrm{s}$ to $50 \mathrm{~m} / \mathrm{s}$, with $10 \mathrm{~m} / \mathrm{s}$ intervals, and the corresponding change in the windage yaw angle is shown in Figure 6. With increasing wind speed, both $\bar{\varphi}$ and $\widehat{\varphi}$ increase nonlinearly, and the rate of increase decreases, indicating that the tangent stiffness of the conductor windage yaw gradually increases. The influence of the mean wind speed on $\beta_{c}$ is shown in Figure 6 and Table 3 . With increasing wind speed, $\beta_{c}$ increases nonlinearly, and the rate of increase gradually becomes larger because of the influence of the stiffness changes.

4.2. Influence of Height Difference between the Two Suspension Points of Conductors. The influence of the height difference is studied by the overall vertical translation of the conductor of the first span on the left in Figure 2. A downward translation means a positive height difference for suspension point 2, with respect to suspension point 1 , whereas an upwards translation means a negative height difference for that point (as shown in Figure 2). The height difference between the target point and the suspension point 1 is between $-60 \mathrm{~m}$ and $20 \mathrm{~m}$. With the decrease in the magnitude of the height difference, the wind loads and the selfweight of the conductors transmitted to the target point of the insulator string increase and decrease, respectively, which, in turn, results in a change in the stress stiffness of the insulator string. The distribution of the windage yaw angle corresponding to different height differences is shown in Figure 7 . With the decreasing height difference, $\bar{\varphi}$ and $\widehat{\varphi}$ gradually increase, and the height difference versus windage yaw angle relationship is approximately linear. The influence of the height difference on $\beta_{c}$ is shown in Figure 7 and Table 4 . As the height difference increases, $\beta_{c}$ first increases, then decreases, and finally, it increases again. $\beta_{\mathrm{c}}$ is larger when there is a height difference than in the case of no height difference. The absolute maximum deviation of $\beta_{c}$ from its average value is $3.2 \%$. Thus, within the range of $h=-60 \mathrm{~m}$ to $20 \mathrm{~m}$, the height difference only slightly affects $\beta_{\mathrm{c}}$.

4.3. Influence of Nominal Height. The nominal height $(H)$ is varied to vertically translate the whole TL from $H=25 \mathrm{~m}$ to $105 \mathrm{~m}$ at the intervals of $20 \mathrm{~m}$. As shown in figure 4(a), as the nominal height increases, the average wind speed increases, and turbulence intensity decreases. The variation of the windage yaw angle with nominal height is shown in Figure 8, showing that as the nominal height increases, $\bar{\varphi}$ and $\widehat{\varphi}$ increase nonlinearly, while the rate of that increase decreases. The influence of the nominal height on $\beta_{c}$ is shown in Figure 8 and Table 5 . Under the combined effect of the average wind speed and turbulence intensity, the nominal height has little influence on $\beta_{c}$, and this influence has no obvious regularity.

4.4. Influence of the Sag-to-Span Ratio. Generally, when the sag-to-span ratio, $\eta$, increases, the wind loads on the conductor decrease. Because of the decrease in the initial tension, the stress stiffness of the conductor also decreases. Hence, the sag-to-span ratio affects the windage yaw characteristics of conductors from two aspects. The variation of windage yaw angle with sag-to-span ratio, presented in Figure 9, shows that as the sag-to-span ratio increases, $\bar{\varphi}$ and $\widehat{\varphi}$ decrease nonlinearly. The influence of the sag-to-span ratio on $\beta_{c}$ is shown in Figure 9 and Table 6 . As the sag-tospan ratio is varied, the trend of $\beta_{c}$ is not obvious. The absolute maximum deviation of $\beta_{c}$ from its average value is $4.7 \%$, and hence, the variability, within the range of $\eta=1 \%$ to $5 \%$, is small.

4.5. Influence of Span. The sag-to-span ratio of the conductor is kept unchanged, and the design span $(550 \mathrm{~m}$, as shown in Figure 2) is varied from $150 \mathrm{~m}$ to $950 \mathrm{~m}$ in the intervals of $200 \mathrm{~m}$. With an increase in span, the spatial correlation of the 


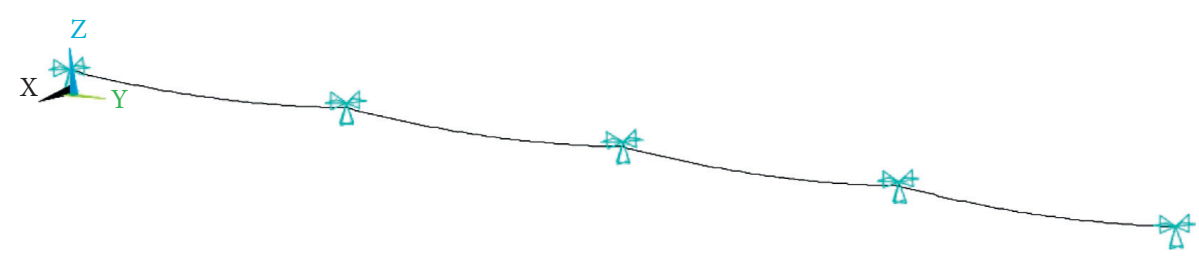

FIGURE 5: Isometric view of the FEM with four-span conductors in ANSYS software.

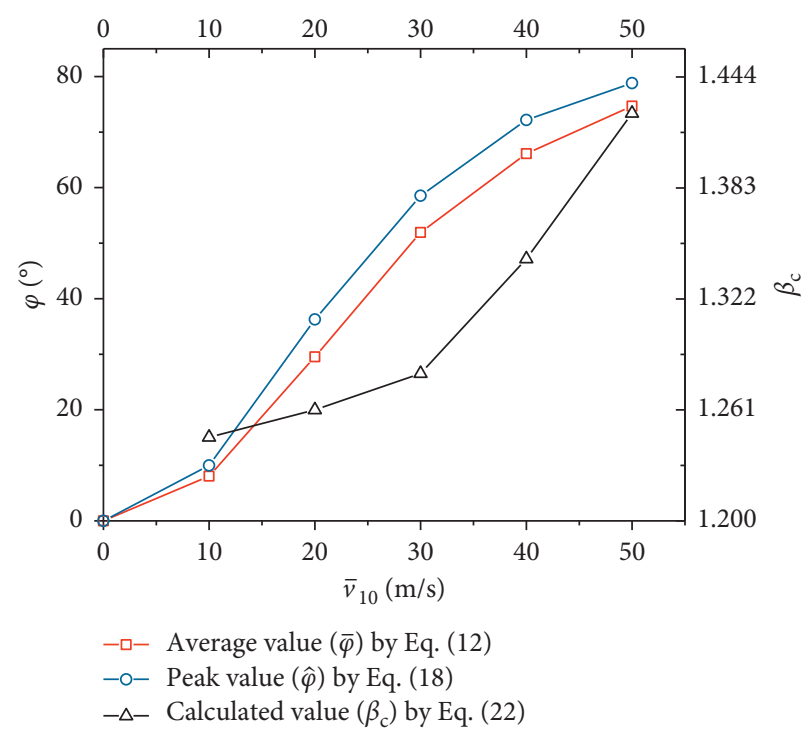

Figure 6: Influence of mean wind speed $\left(\bar{v}_{10}\right)$ on the windage yaw angle $(\varphi)$.

TABLE 3: Influence of wind speed on the dynamic wind load factor of the windage yaw angle.

\begin{tabular}{lccccc}
\hline $\bar{v}_{10}(\mathrm{~m} / \mathrm{s})$ & 10 & 20 & 30 & 40 & 50 \\
\hline$\beta_{\mathrm{c}}$ & 1.246 & 1.261 & 1.281 & 1.344 & 1.424 \\
\hline
\end{tabular}

wind loads decreases, however, the wind loads and selfweight of the conductors increase. The windage yaw angle distribution corresponding to different spans is shown in Figure 10. Under the combined action of the wind loads and self-weight, as the span increases, $\bar{\varphi}$ and $\widehat{\varphi}$ increase first and then decrease. When the span is $550 \mathrm{~m}$, the windage yaw angle reaches its maximum value. The influence of span on $\beta_{\mathrm{c}}$ is shown in Figure 10 and Table 7. Because of the joint influence of the wind loads and self-weight, with the increase in span, $\beta_{c}$ decreases, and the rate of decrease becomes smaller.

4.6. Influence of Ground Roughness. The ground roughness category of the Chinese load code GB 50009-2012 is divided into four categories. Most TLs belong to category B ground roughness because they are used for fields, villages, jungles, hills, and towns with sparse houses. In addition, long-span TLs are in category A ground roughness, which means that they are used for offshore water surfaces and islands. A few TLs that are located in cities belong to category C or D ground roughness, which are for dense urban areas. The design ground roughness case is changed from $\mathrm{A}$ to

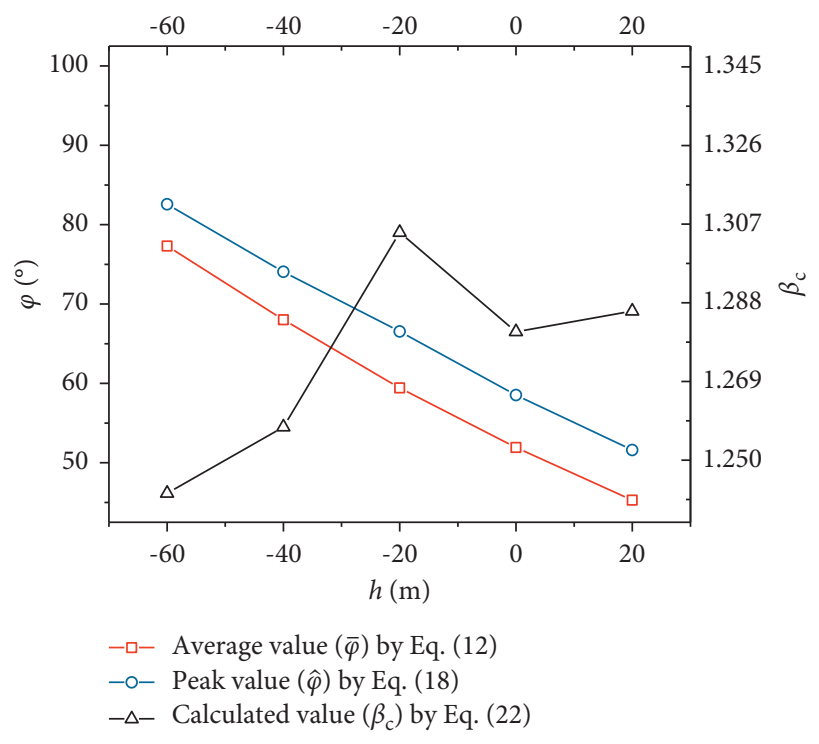

FIGURE 7: Influence of height difference $(h)$ on the windage yaw angle $(\varphi)$.

D. Figure 11 shows that when the roughness category is changed from $\mathrm{A}$ to $\mathrm{D}, \bar{\varphi}$ and $\hat{\varphi}$ decrease. It is because the wind speeds within the conductor height range decrease as roughness increases for a given reference wind speed. The influence of the ground roughness category on $\beta_{c}$ is shown in Figure 11 and Table 8 . As the ground roughness category 
TABLE 4: Influence of height difference on the dynamic wind load factor of the windage yaw angle.

\begin{tabular}{lccccc}
\hline$h(\mathrm{~m})$ & -60 & -40 & -20 & 0 & 20 \\
\hline$\beta_{\mathrm{c}}$ & 1.242 & 1.258 & 1.305 & 1.281 & 1.286 \\
\hline
\end{tabular}

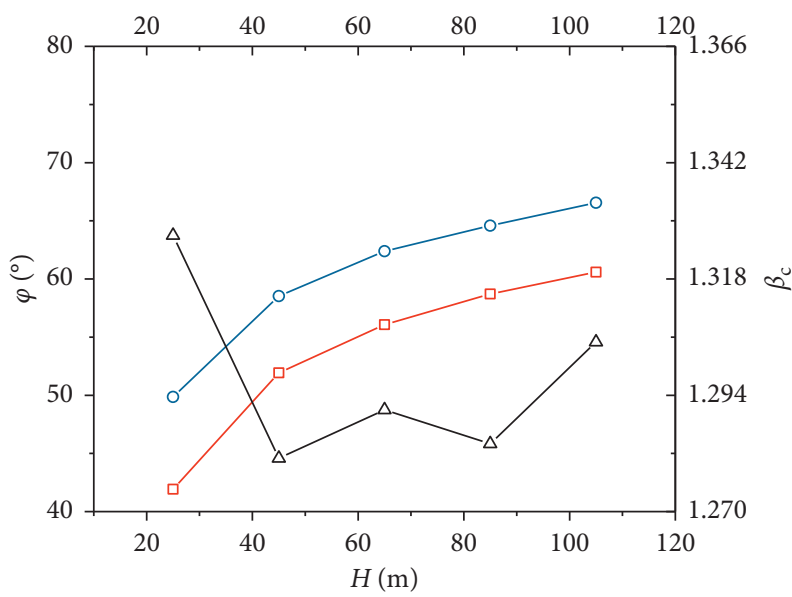

$$
\begin{aligned}
& -\square-\text { Average value }(\bar{\varphi}) \text { by Eq. }(12) \\
& -\circ-\text { Peak value }(\hat{\varphi}) \text { by Eq. }(18) \\
& -\Delta-\text { Calculated value }\left(\beta_{c}\right) \text { by Eq. }(22)
\end{aligned}
$$

Figure 8: Influence of nominal height $(H)$ on the windage yaw angle $(\varphi)$.

TABLE 5: Influence of nominal height on the dynamic wind load factor of the windage yaw angle.

\begin{tabular}{lccccc}
\hline$H(\mathrm{~m})$ & 25 & 45 & 65 & 85 & 105 \\
\hline$\beta_{\mathrm{c}}$ & 1.327 & 1.281 & 1.291 & 1.284 & 1.305 \\
\hline
\end{tabular}

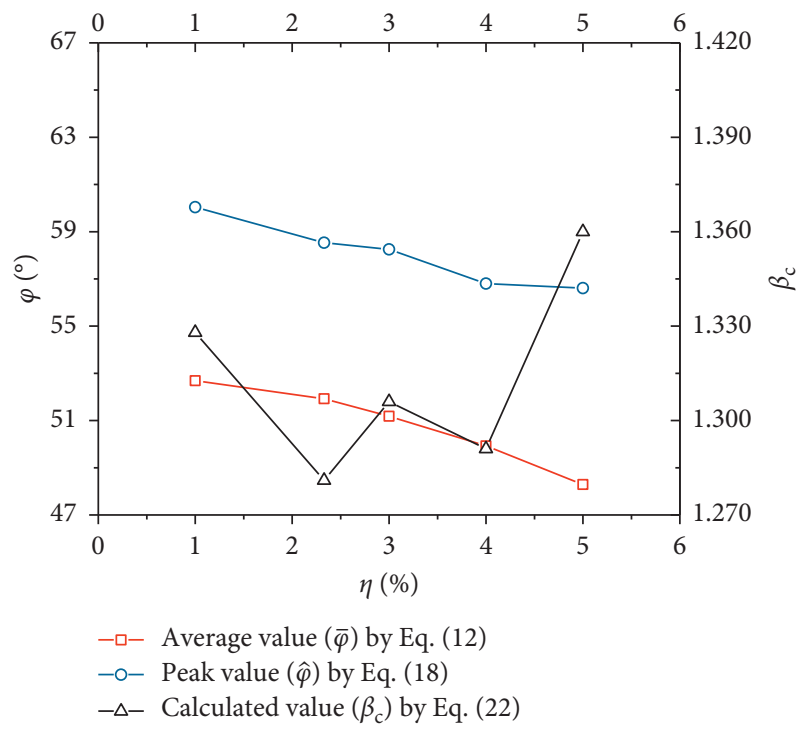

FIGURE 9: Influence of sag-to-span ratio $(\eta)$ on the windage yaw angle $(\varphi)$.
TABLE 6: Influence of sag-to-span ratio on the dynamic wind load factor of the windage yaw angle.

\begin{tabular}{lccccc}
\hline$\eta(\%)$ & 1 & 2.33 & 3 & 4 & 5 \\
\hline$\beta_{c}$ & 1.328 & 1.281 & 1.306 & 1.291 & 1.360 \\
\hline
\end{tabular}

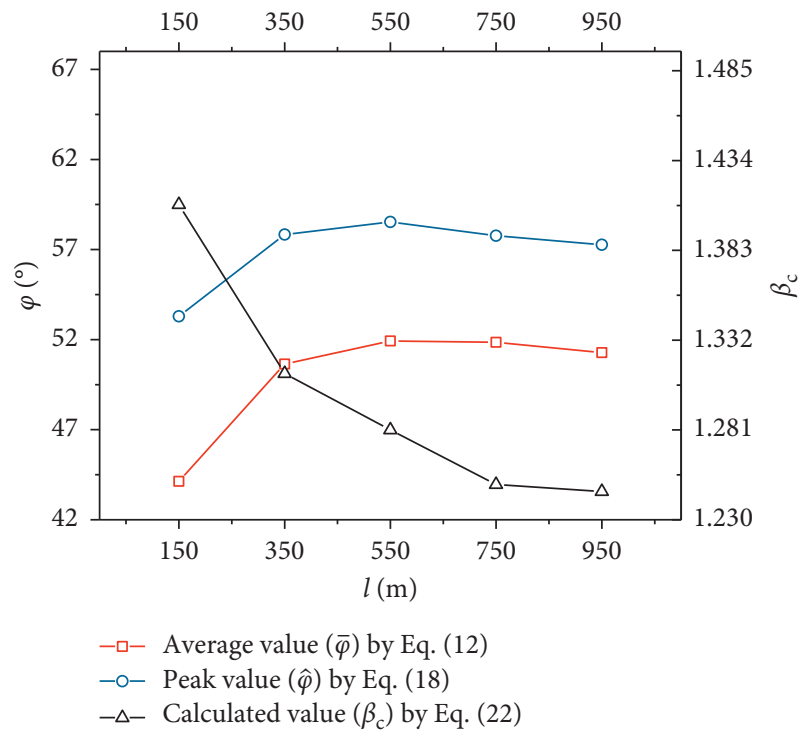

FIGURE 10: Influence of span $(l)$ on the windage yaw angle $(\varphi)$.

TABLE 7: Influence of span on the dynamic wind load factor of the windage yaw angle.

\begin{tabular}{lccccc}
\hline$l(\mathrm{~m})$ & 150 & 350 & 550 & 750 & 950 \\
\hline$\beta_{\mathrm{c}}$ & 1.409 & 1.313 & 1.281 & 1.250 & 1.246
\end{tabular}

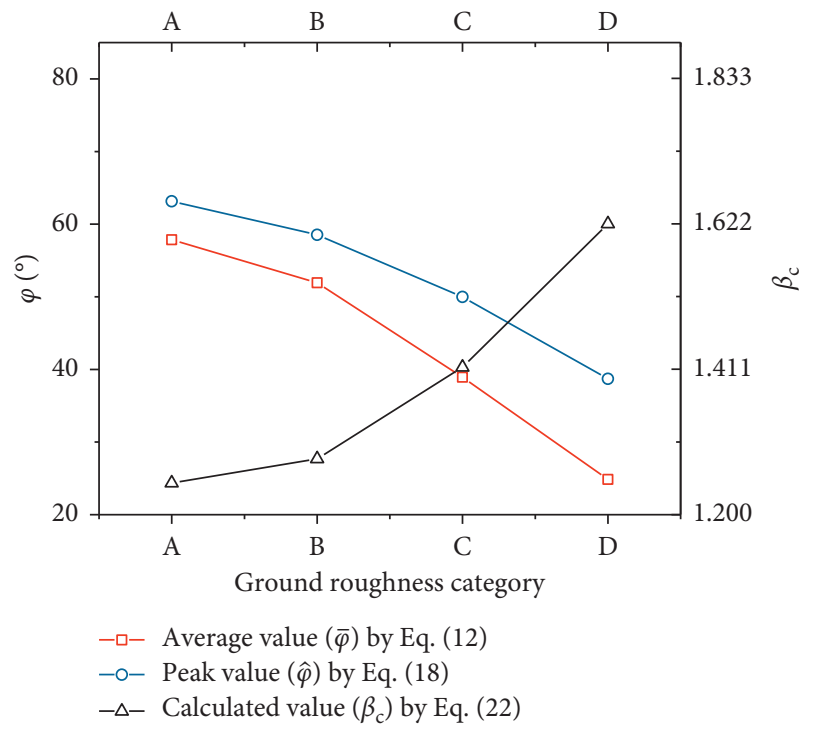

FIGURE 11: Influence of ground roughness category on the windage yaw angle $(\varphi)$. 
TABLE 8: Influence of ground roughness category on the dynamic wind load factor of the windage yaw angle.

\begin{tabular}{lcccc}
\hline Category ground roughness & $A$ & $B$ & $C$ & $D$ \\
\hline$\beta_{\mathrm{c}}$ & 1.246 & 1.281 & 1.414 & 1.622 \\
\hline
\end{tabular}

changes from $A$ to $D, \beta_{c}$ increases. It is because the static wind effect of the conductors decreases, whereas its dynamic wind effect increases, as roughness increases for a given reference wind speed.

\section{Conclusions}

By introducing the rigid-body rule and an improvement to the rigid straight rod (RSR) model, a calculation model was proposed to determine the peak dynamic windage yaw angle $\hat{\varphi}$, together with the dynamic wind load factor $\beta_{c}$, of suspension insulator strings (SISs). The proposed calculation model was then verified by the results from a time-domain calculation. By changing the values of important design parameters within the design range, the influence of these parameters on $\hat{\varphi}$ and $\beta_{c}$ was analyzed. The main findings are summarized as follows:

(i) According to the wind-induced vibration characteristics (large deformation and small amplitude) of the conductors, with the average wind state as the initial calculation condition, the load-responsecorrelation (LRC) method can be used to calculate the equivalent static wind loads (ESWLs) and $\widehat{\varphi}_{0}$ of SISs. The established RSR model improves the calculation accuracy, especially for the case where there is a height difference, and it is suitable for calculating the average windage yaw angle $(\bar{\varphi})$. Compared with the calculation of $\hat{\varphi}$ in the existing method, the proposed method is not only applicable to complex transmission line (TL) scenarios but also computationally more efficient.

(ii) Taking an actual TL as an example, the ESWLs, $\bar{\varphi}, \widehat{\varphi}$, and $\beta_{\mathrm{c}}$ are calculated by the proposed calculation model with regards to the windage yaw of an SIS. The ESWLs reach their maximum value at the selected target point position. $\widehat{\varphi}$ is 1.127 times that of $\bar{\varphi}$, and the corresponding $\beta_{\mathrm{c}}$ is 1.281 , indicating that the dynamic effect and nonlinearity of the windage yaw is obvious and cannot be ignored in the calculation. The accuracy and efficiency of the proposed calculation model are verified by the FEM results.

(iii) For increasing wind speed and nominal height, $\bar{\varphi}$ and $\hat{\varphi}$ increase. For increasing height difference and sag-to-span ratio and when the ground roughness category is changed from A to $\mathrm{D}, \bar{\varphi}$ and $\hat{\varphi}$ decrease. With increasing span, $\bar{\varphi}$ and $\widehat{\varphi}$ initially increase and then decrease, with a span of $550 \mathrm{~m}$ being the most unfavorable. With increasing wind speed and when the ground roughness category is changed from $A$ to $\mathrm{D}, \beta_{\mathrm{c}}$ increases. With increasing span, $\beta_{\mathrm{c}}$ decreases. The height difference, nominal height, and sag-to- span ratio only have a small influence on $\beta_{\mathrm{c}}$ and may be neglected in the approximate calculation of $\beta_{c}$.

(iv) In the presented work, reference has been made specifically to the Chinese code, and only one type of conductor and SIS is selected for conducting the influence parameter analyses. For the application of the present work in TL design using other types of conductors in different countries and regions, more types should ideally be considered, and the methodology proposed here can be used to determine the value of $\beta_{c}$ for the selected conductor in relation to other national or regional codes.

\section{Data Availability}

The source data used in this paper are not generally available; however, the reader may contact the corresponding author for specific information or data.

\section{Conflicts of Interest}

The authors declare that they have no conflicts of interest.

\section{Acknowledgments}

This study was supported by the Science and Technology Research Program of Chongqing Municipal Education Commission (Grant No. KJQN202001548), Scientific Research Foundation of Chongqing University of Science and Technology (Grant No. ckrc2019036), and National Natural Science Foundation of China (Grant No. 51808078).

\section{References}

[1] D. H. Wang, J. Li, and Q. Xie, “Analytical model for dynamic tension in overhead power transmission lines subject to strong wind," Advances in Structural Engineering, vol. 14, no. 3, pp. 445-456, 2011.

[2] D. H. Wang and J. Li, "Wind load in design of long-span transmission lines based on RMS dynamic tension response spectrum," Journal of Vibration and Shock, vol. 31, no. 9, pp. 82-89, 2012.

[3] National Energy Administration, Technical Code for the Design of tower and Pole Structures of Overhead Transmission Lines, National Energy Administration of the People's Republic of China, Beijing, China, 2012, in Chinese.

[4] D. H. Wang, H. Y. Wu, and S. G. Liang, "Theoretical analysis and comparison on typical international wind load codes of transmission conductors," Proceedings of the Chinese Society for Electrical Engineering, vol. 34, no. 36, pp. 6613-6621, 2014.

[5] American Society of Civil Engineers, Guidelines for Electrical Transmission Line Structural Loading, American Society of Civil Engineers, Reston, USA, 4th edition, 2020.

[6] British Standards Institution, Lattice Towers and Masts - Part 1: Code of Practice for Loading, British Standards Institution, London, UK, 1986.

[7] W. J. Lou, Y. Yang, Z. B. Lv, S. F. Zhang, and L. Yang, "Windage yaw dynamic analysis methods for transmission lines considering aerodynamic damping effect," Journal of Vibration and Shock, vol. 34, no. 6, pp. 24-29, 2015.

[8] J. Shao, J. Wang, M. Long, J. Wang, Y. Tang, and M. Sun, "Study on windage yaw calculation and real-time warning 
method of Shanxi power grid considering microclimate and micro-terrain factors," IEEJ Transactions on Electrical and Electronic Engineering, vol. 13, no. 5, pp. 681-688, 2018.

[9] C. Zhou, J. Yin, and Y. Liu, "Large swing behavior of overhead transmission lines under rain-load conditions," Energies, vol. 11, no. 5, Article ID 1092, 2018.

[10] J. Li, D. Mao, Q. Zhu, and Q. Gao, "Research on windage yaw characteristics of high-voltage insulators in complex wind field," IOP Conference Series: Materials Science and Engineering, vol. 892, no. 1, Article ID 012113, 2020.

[11] B. Bo Yan, X. S. Xuesong Lin, W. Wei Luo, Z. D. Zhongquan Liu, and Z. Q. Liu, "Numerical study on dynamic swing of suspension insulator string in overhead transmission line under wind load," IEEE Transactions on Power Delivery, vol. 25, no. 1, pp. 248-259, 2010.

[12] J. C. Wang, S. W. Zhu, B. Peng, S. B. Duan, and P. Li, "Static and dynamic mechanical characteristic comparison research of v-type insulator string under gale condition," IOP Conference Series: Earth and Environmental Science, vol. 61, Article ID 012094, 2017.

[13] L. Q. An, Y. Y. Guan, Z. J. Zhu, and R. L. Zhang, "Research on windage yaw flashovers of transmission lines under wind and rain conditions," Energies, vol. 12, no. 19, p. 3728, 2019.

[14] Y. Z. Jia, M. X. Xiao, and B. You, "The windage yaw numerical simulation of 500-KV overhead transmission lines," in Proceedings of the IEEE International Conference on Power System Technology, Zhejiang, China, October, 2010.

[15] L. Li, L. H. Xiao, X. G. Luo, and X. Min, "Windage yaw calculation method of UHV insulator strings," High Voltage Engineering, vol. 39, no. 12, pp. 2924-2932, 2013.

[16] L. Li, C. L. Lin, and X. Wu, "Research on windage yaw of V-type composite insulators in ultra-high voltage," in Proceedings of the 2nd International Conference on Mechanics, Materials and Structural Engineering, Beijing, China, April, 2017.

[17] Y. B. Yang and H. T. Chiou, "Rigid body motion test for nonlinear analysis with beam elements," Journal of Engineering Mechanics, vol. 113, no. 9, pp. 1404-1419, 1987.

[18] Y. B. Yang and S. R. Kuo, Theory and Analysis of Nonlinear Framed Structures, Prentice-Hall, Singapore, 1994.

[19] A. G. Davenport, "Gust load factors," Journal of the Structural Division - ASCE, vol. 93, no. ST3, pp. 11-34, 1967.

[20] M. Kasperski and H. J. Niemann, "The L.R.C. (load-responsecorrelation)-method a general method of estimating unfavourable wind load distributions for linear and non-linear structural behavior," Journal of Wind Engineering and Industrial Aerodynamics, vol. 43, no. 1-3, pp. 1753-1763, 1992.

[21] J. D. Holmes, "Effective static load distributions in wind engineering," Journal of Wind Engineering and Industrial Aerodynamics, vol. 90, no. 2, pp. 91-109, 2002.

[22] W. J. Lou, G. Luo, and W. K. Hu, "Calculation method for equivalent static wind loads and wind load adjustment coefficients for transmission lines," Journal of Zhejiang University (Engineering Science), vol. 50, no. 11, pp. 2120-2127, 2016.

[23] H. Z. Deng, S. Y. Zhu, and Z. M. Wang, "Dynamic behavior and wind-induced vibration response of long span transmission line system," Building Structure, vol. 34, no. 7, pp. 25-28, 2004.

[24] S. Zhao, Z. T. Yan, Z. L. Li, J. Y. Dong, and X. C. Nie, "Design and analysis of an aeroelastic model for the $1000 \mathrm{kV}$ Sutong long span transmission tower-line system," Journal of $\mathrm{Vi}$ bration and Shock, vol. 38, no. 12, pp. 1-8, 2019.
[25] A. M. Loredo-Souza and A. G. Davenport, "A novel approach for wind tunnel modelling of transmission lines," Journal of Wind Engineering and Industrial Aerodynamics, vol. 89, no. 11-12, pp. 1017-1029, 2001.

[26] M. M. Darwish, A. A. El Damatty, and H. Hangan, "Dynamic characteristics of transmission line conductors and behaviour under turbulent downburst loading," Wind and Structures An International Journal, vol. 13, no. 4, pp. 327-346, 2010.

[27] Ministry of Housing and Urban-Rural Construction, $110 k$ V 750kV Overhead Transmission Line Design Specification, Ministry of Housing and Urban-Rural Construction of the People's Republic of China, Beijing, China, 2010, in Chinese.

[28] Ministry of Housing and Urban-Rural Construction, Load Code for the Design of Building Structures, Ministry of Housing and Urban-Rural Construction of the People's Republic of China, Beijing, China, 2012, in Chinese.

[29] G. Deodatis, "Simulation of ergodic multivariate stochastic processes," Journal of Engineering Mechanics, vol. 122, no. 8, pp. 778-787, 1996.

[30] A. G. Davenport, "The relationship of wind structure to wind loading," in Proceedings of the Conference on Wind Effects on Buildings \& Structures, Teddington, 1965.

[31] M. Shiotani and H. Arai, "Lateral structures of gusts in high winds," in Proceedings of the International Conference on Wind Effects on Buildings and Structures, vol. 1, pp. 20-26, Ottawa, Canada, 1967.

[32] ANSYS, ANSYS Mechanical APDL Structural Analysis Guide, ANSYS Inc., Pittsburgh, PA, USA, 2020.

[33] X. Fu and H.-N. Li, "Uncertainty analysis of the strength capacity and failure path for a transmission tower under a wind load," Journal of Wind Engineering and Industrial Aerodynamics, vol. 173, pp. 147-155, 2018.

[34] S. Zhao, Z. T. Yan, Z. L. Li, J. Y. Dong, and Y. L. Zhong, "Investigation on wind tunnel tests of an aeroelastic model of $1000 \mathrm{kV}$ Sutong long span transmission tower-line system," Proceedings of the Chinese Society for Electrical Engineering, vol. 38, no. 17, pp. 5257-5265, 2018. 